\title{
Nonmonotonic critical temperature in superconductor/ferromagnet bilayers
}

\author{
Ya. V. Fominov, ${ }^{1,2, *}$ N. M. Chtchelkatchev, ${ }^{1, \dagger}$ and A. A. Golubov ${ }^{2, \ddagger}$ \\ ${ }^{1}$ L. D. Landau Institute for Theoretical Physics RAS, 117940 Moscow, Russia \\ ${ }^{2}$ Department of Applied Physics, University of Twente, P.O. Box 217, 7500 AE Enschede, The Netherlands
}

(Received 19 February 2002; published 25 June 2002)

\begin{abstract}
The critical temperature $T_{c}$ of a superconductor/ferromagnet (SF) bilayer can exhibit nonmonotonic dependence on the thickness $d_{f}$ of the F layer. SF systems have been studied for a long time; according to the experimental situation, a "dirty" limit is often considered which implies that the mean free path in the layers is the second smallest spatial scale after the Fermi wavelength. However, all calculations reported for the dirty limit were done with some additional assumptions, which can be violated in actual experiments. Therefore, we develop a general method (to be exact, two independent methods) for investigating $T_{c}$ as a function of the bilayer parameters in the dirty case. Comparing our theory with experiment, we obtain good agreement. In the general case, we observe three characteristic types of $T_{c}\left(d_{f}\right)$ behavior: (1) a nonmonotonic decay of $T_{c}$ to a finite value exhibiting a minimum at particular $d_{f}$; (2) a reentrant behavior, characterized by a vanishing of $T_{c}$ in a certain interval of $d_{f}$ and finite values otherwise; and (3) a monotonic decay of $T_{c}$ and a vanishing at finite $d_{f}$. Qualitatively, the nonmonotonic behavior of $T_{c}\left(d_{f}\right)$ is explained by the interference of quasiparticles in the F layer, which can be either constructive or destructive depending on the value of $d_{f}$.

DOI: 10.1103/PhysRevB.66.014507

PACS number(s): 74.50.+r, 74.80.Dm, 75.30.Et
\end{abstract}

\section{INTRODUCTION}

Superconductivity and ferromagnetism are two competing orders: while the former "prefers" an antiparallel spin orientation of electrons in Cooper pairs, the latter forces the spins to align in parallel. Therefore, their coexistence in one and the same material is possible only in a narrow interval of parameters; hence the interplay between superconductivity and ferromagnetism is most conveniently studied when the two interactions are spatially separated. In this case the coexistence of the two orders is due to the proximity effect. Recently, much attention has been paid to properties of hybrid proximity systems containing superconductors (S's) and ferromagnets (F's); interesting physical phenomena were observed and predicted in these systems. ${ }^{1-6}$ One of the most striking effects in superconductor/ferromagnet (SF)-layered structures is highly nonmonotonic dependence of their critical temperature $T_{c}$ on the thickness $d_{f}$ of the ferromagnetic layers. Experiments exploring this nonmonotonic behavior were performed previously on SF multilayers such as $\mathrm{Nb} / \mathrm{Gd},{ }^{7} \mathrm{Nb} / \mathrm{Fe},{ }^{8} \mathrm{~V} / \mathrm{V}-\mathrm{Fe},{ }^{9}$ and $\mathrm{Pb} / \mathrm{Fe},{ }^{10}$ but the results (and, in particular, the comparison between the experiments and theories) were not conclusive.

To perform reliable experimental measurements of $T_{c}\left(d_{f}\right)$, it is essential to have a large $d_{f}$ compared to the interatomic distance; this situation can be achieved only in the limit of weak ferromagnets. Active experimental investigations of SF bilayers and multilayers based on $\mathrm{Cu}-\mathrm{Ni}$ dilute ferromagnetic alloys were carried out by several groups. ${ }^{11,12}$ In SF bilayers, they observed a nonmonotonic dependence $T_{c}\left(d_{f}\right)$. While the reason for this effect in multilayers can be the 0 - $\pi$ transition, ${ }^{3}$ in a bilayer system with a single superconductor this mechanism is irrelevant, and the cause of the effect is interference of quasiparticle, specific to SF structures.

In the present paper, motivated by the experiments of Refs. 11 and 12, we theoretically study the critical tempera- ture of SF bilayers. Previous theoretical investigations of $T_{c}$ in SF structures were concentrated on systems with thin or thick layers (compared to the corresponding coherence lengths), with SF boundaries having very low or very high transparencies; the exchange energy was often assumed to be much larger than the critical temperature. In addition, the methods for solving the problem were usually approximate. ${ }^{3,4,9,10,13-16}$ The parameters of the experiments of Refs. 11 and 12 did not correspond to any of the above limiting cases. In the present paper we develop two approaches, giving the opportunity to investigate not only the limiting cases of parameters but also the intermediate region. Using our methods, we find different types of nonmonotonic behavior of $T_{c}$ as a function of $d_{f}$, such as a minimum of $T_{c}$ and even reentrant superconductivity. Comparison of our theoretical predictions with the experimental data shows good agreement.

A number of methods can be used for calculating $T_{c}$. When the critical temperature of the structure is close to the critical temperature $T_{c s}$ of a superconductor without a ferromagnetic layer, the Ginzburg-Landau (GL) theory applies. However, $T_{c}$ of SF bilayers may significantly deviate from $T_{c s}$; therefore, we choose a more general theory valid at arbitrary temperature-the quasiclassical approach. ${ }^{17-19}$ Near $T_{c}$ the quasiclassical equations become linear. In the literature the emerging problem is often treated with the help of the so-called "single-mode" approximation, ${ }^{4,14-16}$ which is argued to be qualitatively reasonable in a wide region of parameters. However, this method is justified only in a specific region of parameters which we find below. Moreover, below we show examples when this method fails even qualitatively. Thus there is need for an exact solution of the linearized quasiclassical equations. The limiting case of perfect boundaries and large exchange energy was treated by Radović et al. ${ }^{3}$

Based on the progress achieved for calculations of $T_{c}$ in $\mathrm{SN}$ systems (where $\mathrm{N}$ denotes a nonmagnetic normal 


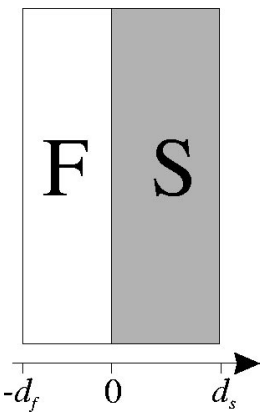

FIG. 1. SF bilayer. The $\mathrm{F}$ and $\mathrm{S}$ layers occupy the regions $-d_{f}<x<0$ and $0<x<d_{s}$, respectively.

material), ${ }^{20}$ we develop a generalization of the single-mode approximation-the multimode method. Although this method seems to be exact, it is subtle to justify it rigorously. Therefore we develop yet another approach (this time mathematically rigorous), which we call "the method of fundamental solution." The models considered previously ${ }^{3,4,9,10,13-16}$ correspond to the limiting cases of our theory. A part of our results was briefly reported in Ref. 21.

The paper is organized as follows. In Sec. II we formulate the Usadel equations and the corresponding boundary conditions. Section III is devoted to the exact multimode method for solving the general equations. An alternative exact method, the method of fundamental solution, is presented in Sec. IV. In Sec. V we describe results of our methods. In Sec. VI, a qualitative explanation of our results is presented, the applicability of the results to multilayered structures is discussed, and the use of a complex diffusion constant is commented upon. Conclusions are presented in Sec. VII. Appendixes A and B contain analytical results for limiting cases. Finally, technical details of the calculations are given in Appendix C.

\section{MODEL}

We assume that the dirty-limit conditions are fulfilled, and calculate the critical temperature of the bilayer within the framework of the linearized Usadel equations for the $\mathrm{S}$ and $\mathrm{F}$ layers (the domain $0<x<d_{s}$ is occupied by the $\mathrm{S}$ metal, and $-d_{f}<x<0$-by the F metal; see Fig. 1). Near $T_{c}$ the normal Green function is $G=\operatorname{sgn} \omega_{n}$, and the Usadel equations for the anomalous function $F$ take the form

$$
\begin{gathered}
\xi_{s}^{2} \pi T_{c s} \frac{d^{2} F_{s}}{d x^{2}}-\left|\omega_{n}\right| F_{s}+\Delta=0, \quad 0<x<d_{s} \\
\xi_{f}^{2} \pi T_{c s} \frac{d^{2} F_{f}}{d x^{2}}-\left(\left|\omega_{n}\right|+i E_{\mathrm{ex}} \operatorname{sgn} \omega_{n}\right) F_{f}=0, \quad-d_{f}<x<0 \\
\Delta \ln \frac{T_{c s}}{T}=\pi T \sum_{\omega_{n}}\left(\frac{\Delta}{\left|\omega_{n}\right|}-F_{s}\right)
\end{gathered}
$$

(the pairing potential $\Delta$ is nonzero only in the $\mathrm{S}$ part). Here $\xi_{s}=\sqrt{D_{s} / 2 \pi T_{c s}}$ and $\xi_{f}=\sqrt{D_{f} / 2 \pi T_{c s}}$ are the coherence lengths, while the diffusion constants can be expressed via the Fermi velocity and the mean free path: $D=v l / 3 ; \omega_{n}$ $=\pi T(2 n+1)$ with $n=0, \pm 1, \pm 2, \ldots$ are the Matsubara frequencies; $E_{\text {ex }}$ is the exchange energy; and $T_{c s}$ is the critical temperature of the $\mathrm{S}$ material. $F_{s(f)}$ denotes the function $F$ in the $S(F)$ region. We use the system of units in which Planck's and Boltzmann's constants equal unity, $\hbar=k_{B}=1$.

Equations (1)-(3) must be supplemented with the boundary conditions at the outer surfaces of the bilayer,

$$
\frac{d F_{s}\left(d_{s}\right)}{d x}=\frac{d F_{f}\left(-d_{f}\right)}{d x}=0,
$$

as well as at the SF boundary: ${ }^{22}$

$$
\begin{gathered}
\xi_{s} \frac{d F_{s}(0)}{d x}=\gamma \xi_{f} \frac{d F_{f}(0)}{d x}, \quad \gamma=\frac{\rho_{s} \xi_{s}}{\rho_{f} \xi_{f}}, \\
\xi_{f} \gamma_{b} \frac{d F_{f}(0)}{d x}=F_{s}(0)-F_{f}(0), \quad \gamma_{b}=\frac{R_{b} \mathcal{A}}{\rho_{f} \xi_{f}} .
\end{gathered}
$$

Here $\rho_{s}$ and $\rho_{f}$ are the normal-state resistivities of the $\mathrm{S}$ and F metals, $R_{b}$ is the resistance of the SF boundary, and $\mathcal{A}$ is its area. The Usadel equation in the F layer is readily solved,

$$
\begin{aligned}
& F_{f}=C\left(\omega_{n}\right) \cosh \left(k_{f}\left[x+d_{f}\right]\right), \\
& k_{f}=\frac{1}{\xi_{f}} \sqrt{\frac{\left|\omega_{n}\right|+i E_{\mathrm{ex}} \operatorname{sgn} \omega_{n}}{\pi T_{c s}}},
\end{aligned}
$$

and the boundary condition at $x=0$ can be written in closed form with respect to $F_{s}$ :

$$
\begin{gathered}
\xi_{s} \frac{d F_{s}(0)}{d x}=\frac{\gamma}{\gamma_{b}+B_{f}\left(\omega_{n}\right)} F_{s}(0), \\
B_{f}=\left[k_{f} \xi_{f} \tanh \left(k_{f} d_{f}\right)\right]^{-1} .
\end{gathered}
$$

This boundary condition is complex. In order to rewrite it in a real form, we do the usual trick and go over to the functions

$$
F^{ \pm}=F\left(\omega_{n}\right) \pm F\left(-\omega_{n}\right) .
$$

According to the Usadel equations (1)-(3), there is a symmetry $F\left(-\omega_{n}\right)=F^{*}\left(\omega_{n}\right)$ which implies that $F^{+}$is real while $F^{-}$is a purely imaginary function.

The symmetric properties of $F^{+}$and $F^{-}$with respect to $\omega_{n}$ are trivial, so we shall treat only positive $\omega_{n}$. The selfconsistency equation is expressed only via the symmetric function $F_{s}^{+}$,

$$
\Delta \ln \frac{T_{c s}}{T}=\pi T \sum_{\omega_{n}>0}\left(\frac{2 \Delta}{\omega_{n}}-F_{s}^{+}\right),
$$

and the problem of determining $T_{c}$ can be formulated in a closed form with respect to $F_{s}^{+}$as follows. The Usadel equation for the antisymmetric function $F_{s}^{-}$does not contain $\Delta$; hence it can be solved analytically. After that we exclude $F_{s}^{-}$ from boundary condition (8) and arrive at the effective boundary conditions for $F_{s}^{+}$, 


$$
\xi_{s} \frac{d F_{s}^{+}(0)}{d x}=W\left(\omega_{n}\right) F_{s}^{+}(0), \quad \frac{d F_{s}^{+}\left(d_{s}\right)}{d x}=0
$$

where

$$
\begin{gathered}
W\left(\omega_{n}\right)=\gamma \frac{A_{s}\left(\gamma_{b}+\operatorname{Re} B_{f}\right)+\gamma}{A_{s}\left|\gamma_{b}+B_{f}\right|^{2}+\gamma\left(\gamma_{b}+\operatorname{Re} B_{f}\right)}, \\
A_{s}=k_{s} \xi_{s} \tanh \left(k_{s} d_{s}\right), \quad k_{s}=\frac{1}{\xi_{s}} \sqrt{\frac{\omega_{n}}{\pi T_{c s}}} .
\end{gathered}
$$

The self-consistency equation (10) and boundary conditions (11) and (12), together with the Usadel equation for $F_{s}^{+}$,

$$
\xi_{s}^{2} \pi T_{c s} \frac{d^{2} F_{s}^{+}}{d x^{2}}-\omega_{n} F_{s}^{+}+2 \Delta=0
$$

will be used below for finding the critical temperature of the bilayer.

The problem can be solved analytically only in limiting cases (see Appendix A). In the general case, one should use a numerical method, and below we propose two methods for solving the problem exactly.

\section{MULTIMODE METHOD}

\section{A. Starting point: the single-mode approximation and its applicability}

In the single-mode approximation (SMA) one seeks a solution of problem (10)-(13) in the form

$$
\begin{gathered}
F_{s}^{+}\left(x, \omega_{n}\right)=f\left(\omega_{n}\right) \cos \left(\Omega \frac{x-d_{s}}{\xi_{s}}\right), \\
\Delta(x)=\delta \cos \left(\Omega \frac{x-d_{s}}{\xi_{s}}\right) .
\end{gathered}
$$

This anzatz automatically satisfies boundary condition (11) at $x=d_{s}$.

The Usadel equation (13) yields

$$
f\left(\omega_{n}\right)=\frac{2 \delta}{\omega_{n}+\Omega^{2} \pi T_{c s}},
$$

then the self-consistency equation (10) takes the form ( $\delta$ and $\Omega$ do not depend on $\omega_{n}$ )

$$
\ln \frac{T_{c s}}{T_{c}}=\psi\left(\frac{1}{2}+\frac{\Omega^{2}}{2} \frac{T_{c s}}{T_{c}}\right)-\psi\left(\frac{1}{2}\right),
$$

where $\psi$ is the digamma function.

Boundary condition (11) at $x=0$ yields

$$
\Omega \tan \left(\Omega \frac{d_{s}}{\xi_{s}}\right)=W\left(\omega_{n}\right) .
$$

The critical temperature $T_{c}$ is determined by Eqs. (17) and (18).

Although this method is popular, it is often used without pointing out the limits of its applicability. We present the explicit formulation of the corresponding condition: the single-mode method is correct only if the parameters are such that $W$ can be considered $\omega_{n}$ independent [because the left-hand side of Eq. (18) must be $\omega_{n}$-independent]. ${ }^{13}$

Appendix B demonstrates examples of the SMA validity and corresponding analytical results. In one of experimentally relevant cases, $E_{\mathrm{ex}} / \pi T_{c s}>1$ and $d_{f} \sim \xi_{f}$, the SMA is applicable if $\sqrt{E_{\mathrm{ex}} / \pi T_{c s}} \gg 1 / \gamma_{b}$ (see Appendix B for details).

\section{B. Inclusion of other modes}

The single-mode approximation implies that one takes the (only) real root $\Omega$ of Eq. (17). An exact (multimode) method for solving problem (10)-(13) is obtained if we also take imaginary roots into account-there is infinite number of these. $^{20}$

Thus we seek the solution in the form

$$
\begin{aligned}
& F_{s}^{+}\left(x, \omega_{n}\right)= f_{0}\left(\omega_{n}\right) \cos \left(\Omega_{0} \frac{x-d_{s}}{\xi_{s}}\right) \\
&+\sum_{m=1}^{\infty} f_{m}\left(\omega_{n}\right) \frac{\cosh \left(\Omega_{m} \frac{x-d_{s}}{\xi_{s}}\right)}{\cosh \left(\Omega_{m} \frac{d_{s}}{\xi_{s}}\right)}, \\
& \Delta(x)=\delta_{0} \cos \left(\Omega_{0} \frac{x-d_{s}}{\xi_{s}}\right)+\sum_{m=1}^{\infty} \delta_{m} \frac{\cosh \left(\Omega_{m} \frac{x-d_{s}}{\xi_{s}}\right)}{\cosh \left(\Omega_{m} \frac{d_{s}}{\xi_{s}}\right)} .
\end{aligned}
$$

(The normalizing denominators in the cosh terms have been introduced in order to increase accuracy of numerical calculations.) This anzatz automatically satisfies boundary condition (11) at $x=d_{s}$.

Substituting the anzatz [Eqs. (19) and (20)] into the Usadel equation (13), we obtain

$$
\begin{gathered}
f_{0}\left(\omega_{n}\right)=\frac{2 \delta_{0}}{\omega_{n}+\Omega_{0}^{2} \pi T_{c s}}, \\
f_{m}\left(\omega_{n}\right)=\frac{2 \delta_{m}}{\omega_{n}-\Omega_{m}^{2} \pi T_{c s}}, \quad m=1,2, \ldots,
\end{gathered}
$$

then the parameters $\Omega$ are determined by the self-consistency equation (10) ( $\delta$ and $\Omega$ do not depend on $\omega_{n}$ ):

$$
\begin{gathered}
\ln \frac{T_{c s}}{T_{c}}=\psi\left(\frac{1}{2}+\frac{\Omega_{0}^{2}}{2} \frac{T_{c s}}{T_{c}}\right)-\psi\left(\frac{1}{2}\right), \\
\ln \frac{T_{c s}}{T_{c}}=\psi\left(\frac{1}{2}-\frac{\Omega_{m}^{2}}{2} \frac{T_{c s}}{T_{c}}\right)-\psi\left(\frac{1}{2}\right), \quad m=1,2, \ldots
\end{gathered}
$$

From Eqs. (22) and properties of the digamma function ${ }^{23}$ it follows that the parameters $\Omega$ belong to the following intervals: 


$$
\begin{gathered}
0<\Omega_{0}^{2}<\frac{1}{2 \gamma_{E}}, \\
\frac{T_{c}}{T_{c s}}(2 m-1)<\Omega_{m}^{2}<\frac{T_{c}}{T_{c s}}(2 m+1), \quad m=1,2, \ldots,
\end{gathered}
$$

where $\gamma_{E} \approx 1.78$ is Euler's constant.

Boundary condition (11) at $x=0$ yields the following equation for the amplitudes $\delta$ :

$$
\begin{aligned}
& \delta_{0} \frac{W\left(\omega_{n}\right) \cos \left(\Omega_{0} d_{s} / \xi_{s}\right)-\Omega_{0} \sin \left(\Omega_{0} d_{s} / \xi_{s}\right)}{\omega_{n}+\Omega_{0}^{2} \pi T_{c s}} \\
&+\sum_{m=1}^{\infty} \delta_{m} \frac{W\left(\omega_{n}\right)+\Omega_{m} \tanh \left(\Omega_{m} d_{s} / \xi_{s}\right)}{\omega_{n}-\Omega_{m}^{2} \pi T_{c s}}=0 .
\end{aligned}
$$

The critical temperature $T_{c}$ is determined by Eqs. (22) and the condition that Eq. (24) has a nontrivial ( $\omega_{n}$ independent) solution with respect to $\delta$.

Numerically, we take a finite number of modes: $m$ $=0,1, \ldots, M$. To take account of $\omega_{n}$ independence of the solution, we write down Eq. (24) at the Matsubara frequencies up to the $N$ th frequency: $n=0,1, \ldots, N$. Thus we arrive at the matrix equation $K_{n m} \delta_{m}=0$ with the following matrix $\hat{K}$ :

$$
\begin{gathered}
K_{n 0}=\frac{W\left(\omega_{n}\right) \cos \left(\Omega_{0} d_{s} / \xi_{s}\right)-\Omega_{0} \sin \left(\Omega_{0} d_{s} / \xi_{s}\right)}{\omega_{n} / \pi T_{c s}+\Omega_{0}^{2}}, \\
K_{n m}=\frac{W\left(\omega_{n}\right)+\Omega_{m} \tanh \left(\Omega_{m} d_{s} / \xi_{s}\right)}{\omega_{n} / \pi T_{c s}-\Omega_{m}^{2}}, \\
n=0,1, \ldots, N, \quad m=1,2, \ldots, M .
\end{gathered}
$$

We take $M=N$, then the condition that Eq. (24) has a nontrivial solution takes the form

$$
\operatorname{det} \hat{K}=0 .
$$

Thus the critical temperature $T_{c}$ is determined as the largest solution of Eqs. (22) and (26).

\section{METHOD OF FUNDAMENTAL SOLUTION}

By definition, the fundamental solution $G\left(x, y ; \omega_{n}\right)$ (which is also called the Green function) of problem (11)(13) satisfies the same equations, but with the deltafunctional "source,", 24

$$
\begin{gathered}
\xi_{s}^{2} \pi T_{c s} \frac{d^{2} G(x, y)}{d x^{2}}-\omega_{n} G(x, y)=-\delta(x-y), \\
\xi_{s} \frac{d G(0, y)}{d x}=W\left(\omega_{n}\right) G(0, y), \quad \frac{d G\left(d_{s}, y\right)}{d x}=0 .
\end{gathered}
$$

The fundamental solution can be expressed via solutions $v_{1}$ and $v_{2}$ of Eq. (27) without the delta function, satisfying the boundary conditions at $x=0$ and $d_{s}$, respectively:

$$
\begin{aligned}
G\left(x, y ; \omega_{n}\right)= & \frac{k_{s} / \omega_{n}}{\sinh \left(k_{s} d_{s}\right)+\left(W / k_{s} \xi_{s}\right) \cosh \left(k_{s} d_{s}\right)} \\
& \times \begin{cases}v_{1}(x) v_{2}(y), & x \leqslant y \\
v_{2}(x) v_{1}(y), & y \leqslant x\end{cases}
\end{aligned}
$$

where

$$
\begin{gathered}
v_{1}(x)=\cosh \left(k_{s} x\right)+\left(W / k_{s} \xi_{s}\right) \sinh \left(k_{s} x\right), \\
v_{2}(x)=\cosh \left(k_{s}\left[x-d_{s}\right]\right) .
\end{gathered}
$$

Having found $G\left(x, y ; \omega_{n}\right)$, we can write the solution of Eqs. (11)-(13) as

$$
F_{s}^{+}\left(x ; \omega_{n}\right)=2 \int_{0}^{d_{s}} G\left(x, y ; \omega_{n}\right) \Delta(y) d y .
$$

Substituting this into the self-consistency equation (10), we obtain

$$
\Delta(x) \ln \frac{T_{c s}}{T_{c}}=2 \pi T_{c} \sum_{\omega_{n}>0}\left[\frac{\Delta(x)}{\omega_{n}}-\int_{0}^{d_{s}} G\left(x, y ; \omega_{n}\right) \Delta(y) d y\right] .
$$

This equation can be expressed in an operator form: $\Delta \ln \left(T_{c s} / T_{c}\right)=\hat{L} \Delta$. Then the condition that Eq. (32) has a nontrivial solution with respect to $\Delta$ is expressed by the equation

$$
\operatorname{det}\left(\hat{L}-\hat{1} \ln \frac{T_{c s}}{T_{c}}\right)=0 .
$$

The critical temperature $T_{c}$ is determined as the largest solution of this equation. Numerically, we put problem (32) and (33) on a spatial grid, so that the linear operator $\hat{L}$ becomes a finite matrix.

\section{NUMERICAL RESULTS}

In Secs. III and IV we developed two methods for calculating the critical temperature of a SF bilayer. Specifying parameters of the bilayer we can find the critical temperature numerically. It can be checked that the multimode method and the method of fundamental solution yield equivalent results. However, at small temperatures $T_{c} \ll T_{c s}$, the calculation time for the multimode method increases. Indeed, the size of the matrix $\hat{K}$ [Eq. (25)] is determined by the number $N$ of the maximum Matsubara frequency $\omega_{N}$, which must be much larger than the characteristic energy $\pi T_{c s}$; hence $N$ $\gg T_{c s} / T_{c}$. Therefore, at low temperatures we use the method of fundamental solution.

\section{A. Comparison with experiment}

Using our methods we fit the experimental data of Ref. 11; the result is presented in Fig. 2. Estimating the parameters $d_{s}=11 \mathrm{~nm}, \quad T_{c s}=7 \mathrm{~K}, \quad \rho_{s}=7.5 \mu \Omega \mathrm{cm}, \quad \xi_{s}$ $=8.9 \mathrm{~nm}, \rho_{f}=60 \mu \Omega \mathrm{cm}, \xi_{f}=7.6 \mathrm{~nm}$, and $\gamma=0.15$ from the experiment, ${ }^{25}$ and fitting only $E_{\text {ex }}$ and $\gamma_{b}$, we find good agreement between our theoretical predictions and the experimental data. 


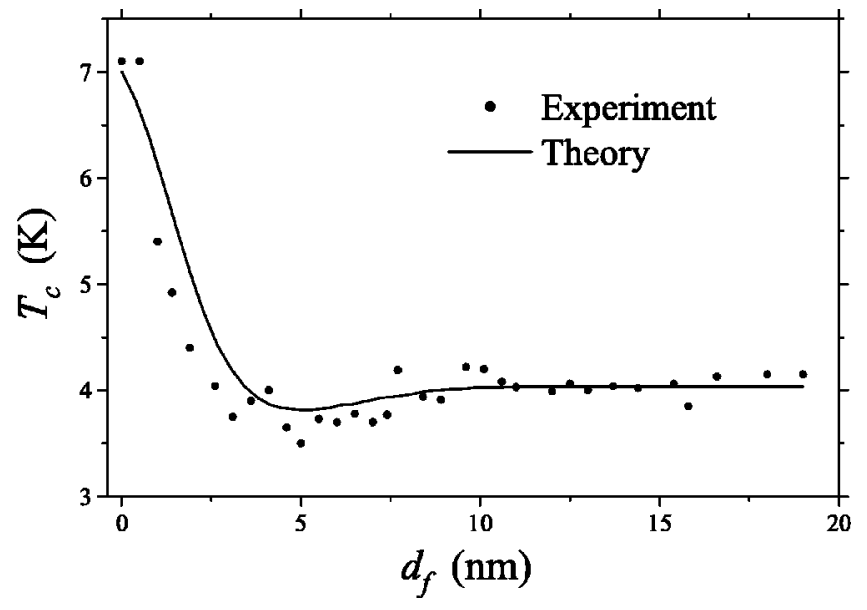

FIG. 2. Theoretical fit to the experimental data of Ref. 11. In the experiment, $\mathrm{Nb}$ was the superconductor (with $d_{s}=11 \mathrm{~nm}, T_{c s}$ $=7 \mathrm{~K}$ ) and $\mathrm{Cu}_{0.43} \mathrm{Ni}_{0.57}$ was the weak ferromagnet. From our fit we estimate $E_{\mathrm{ex}} \approx 130 \mathrm{~K}$ and $\gamma_{b} \approx 0.3$.

The fitting procedure was the following: first, we determine $E_{\mathrm{ex}} \approx 130 \mathrm{~K}$ from the position of the minimum of $T_{c}\left(d_{f}\right)$; second, we find $\gamma_{b} \approx 0.3$ from fitting the vertical position of the curve. The deviation of our curve from the experimental points is small; it is most pronounced in the region of small $d_{f}$ corresponding to the initial decrease of $T_{c}$. This is not unexpected because, when $d_{f}$ is of the order of a few nanometers, the thickness of the F film may vary significantly along the film (which is not taken into account in our theory), and the thinnest films can even be formed by an array of islands rather than by continuous material. At the same time, we emphasize that the minimum of $T_{c}$ takes place at $d_{f} \approx 5 \mathrm{~nm}$, when with good accuracy the $\mathrm{F}$ layer has uniform thickness.

\section{B. Various types of $T_{c}\left(d_{f}\right)$ behavior}

The experimental results discussed above represent only one possible type of $T_{c}\left(d_{f}\right)$ behavior. Now we address the general case; we obtain different kinds of $T_{c}\left(d_{f}\right)$ curves depending on parameters of the bilayer. To illustrate, in Fig. 3 we plot several curves for various values of $\gamma_{b}$ [we recall that $\gamma_{b} \propto R_{b}$, where $R_{b}$ is the resistance of the SF interface in the normal state-see Eq. (6)]. The exchange energy is $E_{\text {ex }}$ $=150 \mathrm{~K}$; the other parameters are the same as in Fig. 2.

We observe three characteristic types of $T_{c}\left(d_{f}\right)$ behavior: (1) At a large enough interface resistance, $T_{c}$ decays nonmonotonically to a finite value exhibiting a minimum at a particular $d_{f}$. (2) At a moderate interface resistance, $T_{c}$ demonstrates a reentrant behavior: it vanishes in a certain interval of $d_{f}$, and is finite otherwise. (3) At a low enough interface resistance $T_{c}$ decays monotonically, vanishing at finite $d_{f}$. A similar succession of $T_{c}\left(d_{f}\right)$ curves as in Fig. 3 can be obtained by tuning other parameters, e.g., the exchange energy $E_{\mathrm{ex}}$ or the normal resistances of the layers (the parameter $\gamma$ ).

A common feature seen from Fig. 3 is saturation of $T_{c}$ at large $d_{f} \gtrsim \lambda_{\text {ex }}$. This fact has a simple physical explanation: the suppression of superconductivity by a dirty ferromagnet

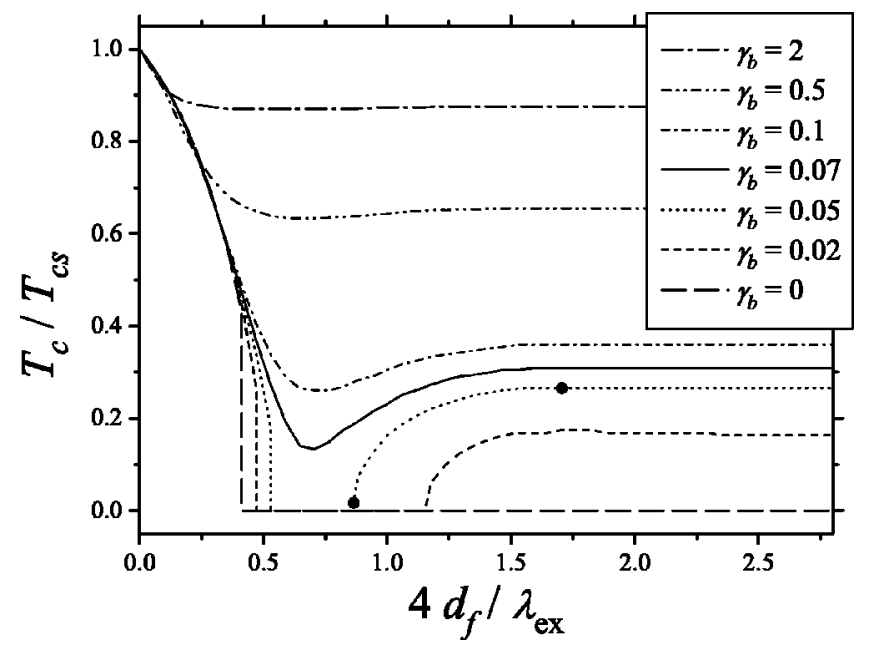

FIG. 3. Characteristic types of $T_{c}\left(d_{f}\right)$ behavior. The thickness of the F layer is measured in units of the wavelength $\lambda_{\mathrm{ex}}$ defined in Eq. (40). The curves correspond to different values of $\gamma_{b}$. The exchange energy is $E_{\text {ex }}=150 \mathrm{~K}$; the other parameters are the same as in Fig. 2. One can distinguish three characteristic types of $T_{c}\left(d_{f}\right)$ behavior: (1) a nonmonotonic decay to a finite $T_{c}$ with a minimum at particular $d_{f}\left(\gamma_{b}=2 ; 0.5 ; 0.1 ; 0.07\right),(2)$ a reentrant behavior $\left(\gamma_{b}=0.05 ; 0.02\right)$, and (3) a monotonic decay to $T_{c}=0$ at finite $d_{f}\left(\gamma_{b}=0\right)$. The bold points indicate the choice of parameter corresponding to Fig. 6.

is only due to the effective $\mathrm{F}$ layer with a thickness on the order of $\lambda_{\mathrm{ex}}$, adjacent to the interface (this is the layer explored and "felt" by quasiparticles entering from the $\mathrm{S}$ side due to the proximity effect).

It was shown by Radovic et al. ${ }^{3}$ that the order of the phase transition may change in short-periodic SF superlattices, becoming of first order. We also observe this feature in the curves of types (2) and (3) mentioned above. This phenomenon manifests itself as discontinuity of $T_{c}\left(d_{f}\right)$ : the critical temperature jumps to zero abruptly without taking intermediate values (see Figs. 3 and 4). Formally, $T_{c}$ becomes a double-valued function, but the smaller solution is physically unstable (dotted curve in Fig. 4).

An interesting problem is determination of the tricritical point where the order of the phase transition changes. The corresponding result for homogeneous bulk superconductors with internal exchange field was obtained a long time ago in the framework of the Ginzburg-Landau (GL) theory. ${ }^{26}$ However, the generalization to the case when the GL theory is not valid is a subtle problem which has not yet been solved. We note that the equations used in Refs. 3 and 15 were applied beyond their applicability range because they are GL results valid only when $T_{c}$ is close to $T_{c s}$.

\section{Comparison between single-mode and multimode methods}

A popular method widely used in the literature for calculating the critical temperature of SF bilayers and multilayers is the single-mode approximation. The condition of its validity was formulated in Sec. III A. However, this approximation is often used for arbitrary system parameters. Using the 


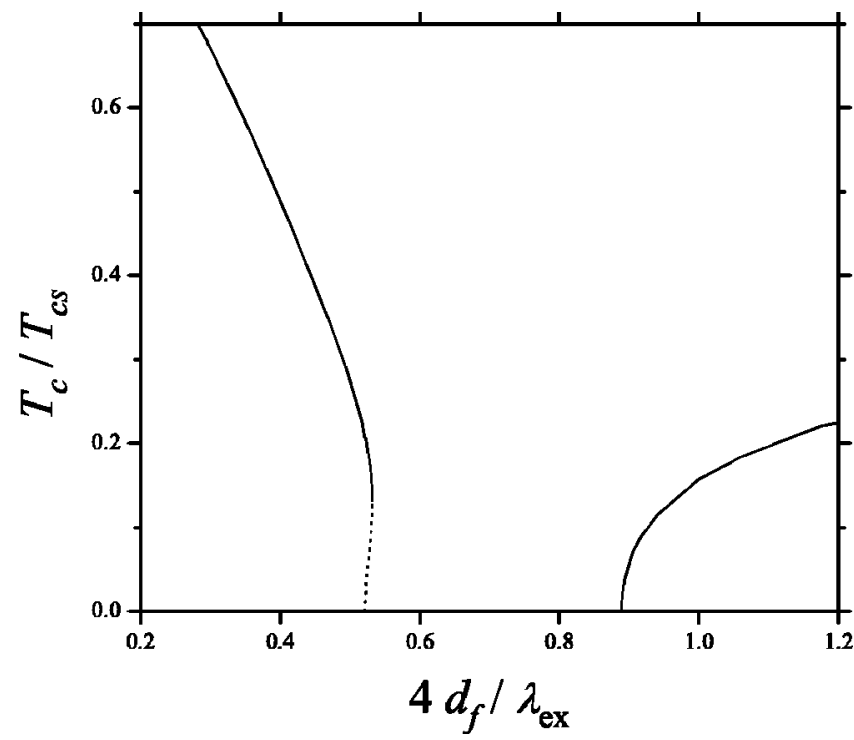

FIG. 4. Change of the phase transition's order. This phenomenon manifests itself as discontinuity of $T_{c}\left(d_{f}\right)$ : the critical temperature jumps to zero abruptly without taking intermediate values. Formally, $T_{c}$ becomes a double-valued function, but the smaller solution is physically unstable (dotted curve). For illustration we have chosen the curve from Fig. 3 corresponding to $\gamma_{b}=0.05$.

methods developed in Secs. III and IV, we can check the actual accuracy of the single-mode approximation. The results are presented in Fig. 5.

We conclude that although at some parameters the results of the single-mode and multi-mode (exact) methods are close [Figs. 5(a) and 5(f)], in the general case they are quantitatively and even qualitatively different [Figs. 5(b)-5(e)these cases correspond to the most nontrivial $T_{c}\left(d_{f}\right)$ behavior]. Thus to obtain reliable results one should use one of the exact (multimode or fundamental-solution) techniques.

\section{Spatial dependence of the order parameter}

The proximity effect in the SF bilayer is characterized by the spatial behavior or the order parameter, which can be chosen as

$$
F(x, \tau=0)=T \sum_{\omega_{n}} F\left(x, \omega_{n}\right),
$$

where $\tau$ denotes the imaginary time [in the $\mathrm{S}$ metal $F(x, \tau$ $=0) \propto \Delta(x)$ ]. This function is real due to the symmetry relation $F\left(-\omega_{n}\right)=F^{*}\left(\omega_{n}\right)$.

We illustrate this dependence in Fig. 6, which shows two cases differing by the thickness of the F layer $d_{f}$ (and by the corresponding $T_{c}$ ). Although the critical temperatures differ by more than the order of magnitude, the normalized order parameters are very close to each other, which means that the value of $T_{c}$ has almost no effect on the shape of $F(x, \tau$ $=0$ ). Details of the calculation are presented in Appendix C.

Another feature seen from Fig. 6 is that the order parameter in the $\mathrm{F}$ layer changes its sign when the thickness of the F layer increases (this feature can be seen for the dotted

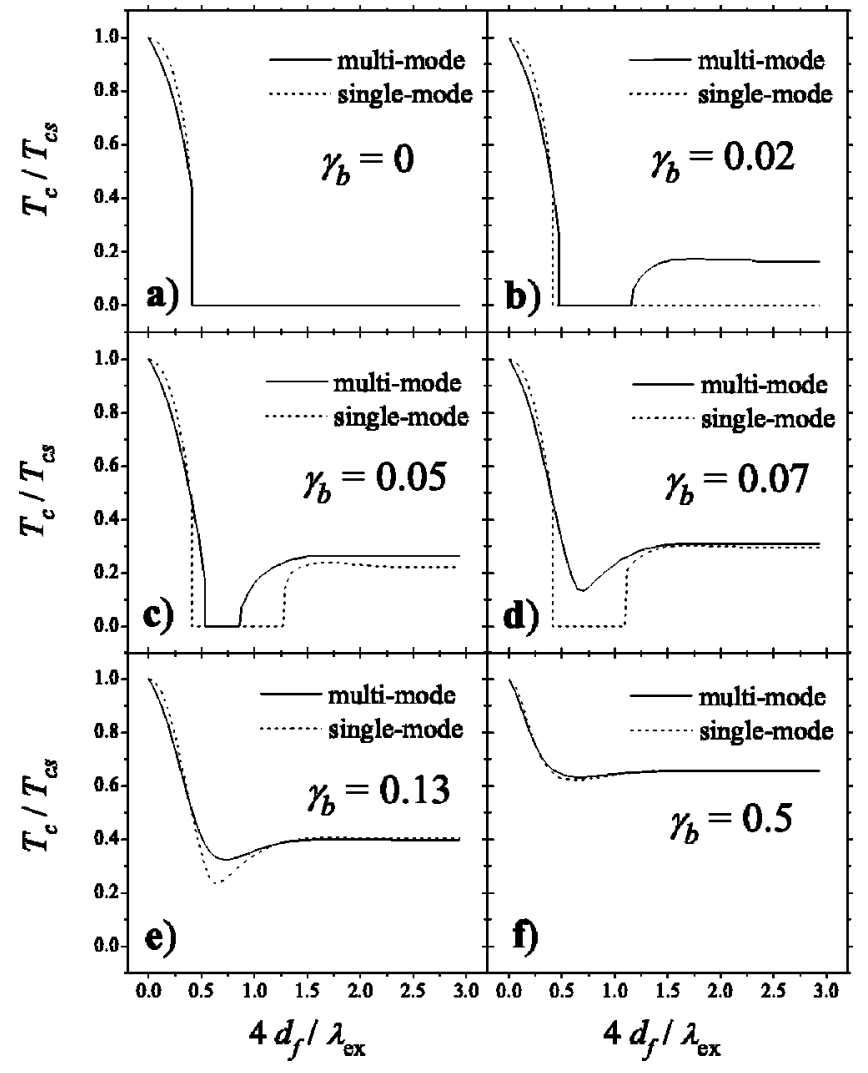

FIG. 5. Comparison between single- and multimode methods. The parameters are the same as in Fig. 3. Generally speaking, the results of the single-mode and multimode (exact) methods are quantitatively and even qualitatively different: (b), (c), (d), and (e). However, sometimes the results are close: (a) and (f). Thus the single-mode approximation can be used for quick estimates, but reliable results should be obtained by one of the exact (multimode or fundamental-solution) techniques.

curve, although negative values of the order parameter have very small amplitudes). We discuss this oscillating behavior in Sec. VI A.

\section{DISCUSSION}

\section{A. Qualitative explanation of the nonmonotonic $\boldsymbol{T}_{c}\left(d_{f}\right)$ behavior}

The thickness of the F layer, at which the minimum of $T_{c}\left(d_{f}\right)$ occurs, can be estimated from qualitative arguments based on the interference of quasiparticles in the ferromagnet. Let us consider a point $x$ inside the $\mathrm{F}$ layer. According to Feynman's interpretation of quantum mechanics, ${ }^{27}$ the quasiparticle wave function may be represented as a sum of wave amplitudes over all classical trajectories; the wave amplitude for a given trajectory is equal to $\exp (i S)$, where $S$ is the classical action along this trajectory. We are interested in an anomalous wave function of correlated quasiparticles, which characterizes superconductivity; this function is equivalent to the anomalous Green function $F(x)$. To obtain this wave function we must sum over trajectories that (i) start and end at the point $x$, and (ii) change the type of the quasiparticle (i.e., convert an electron into a hole, or vice versa). 


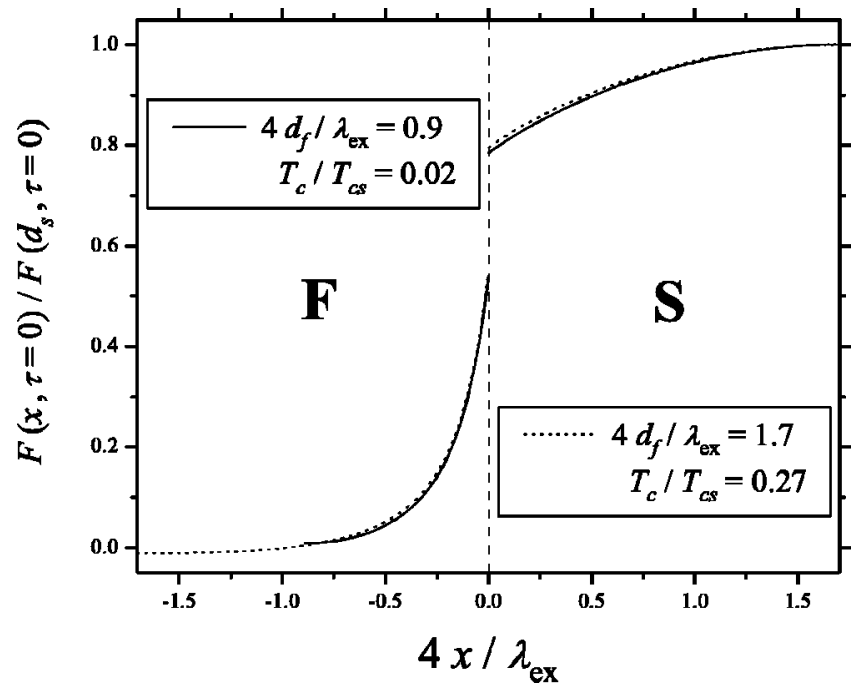

FIG. 6. Spatial dependence of the order parameter normalized by its value at the outer surface of the $\mathrm{S}$ layer. Two cases are shown differing by the thickness of the $\mathrm{F}$ layer $d_{f}$ (and by the corresponding $T_{c}$ ) at $\gamma_{b}=0.05$. The other parameters are the same as in Fig. 3, where the chosen cases are indicated by the bold points. Although the critical temperatures differ by more than the order of magnitude, the normalized order parameters are very close to each other, which means that the value of $T_{c}$ has almost no effect on the shape of $F(x, \tau=0)$. The jump at the SF interface is due to its finite resistance. With an increase of $d_{f}$ the order parameter starts to oscillate, changing its sign (this can be seen for the dotted curve, although negative values of the order parameter have very small amplitudes).

There are four kinds of trajectories that should be taken into account (see Fig. 7). Two of them (denoted 1 and 2) start in the direction toward the SF interface (as an electron and as a hole), experience the Andreev reflection, and return to the point $x$. The other two trajectories (denoted 3 and 4) start in the direction away from the interface, experience normal reflection at the outer surface of the $\mathrm{F}$ layer, move toward the $\mathrm{SF}$ interface, experience the Andreev reflection there, and finally return to the point $x$. The main contribution is given by the trajectories normal to the interface. The corresponding actions are

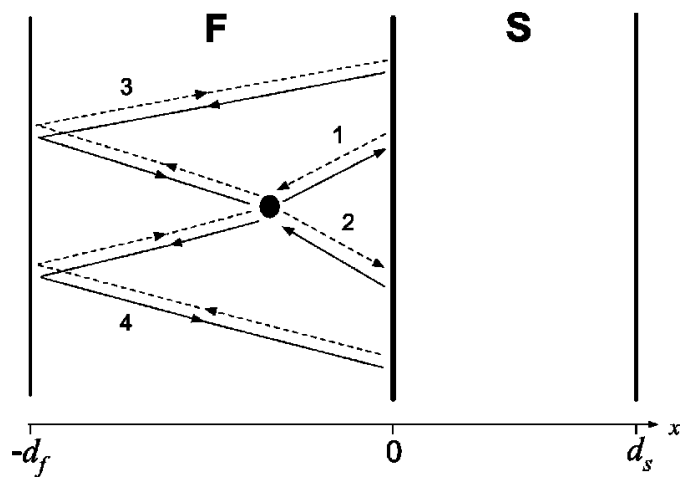

FIG. 7. Four types of trajectories contributing (in the sense of Feynman's path integral) to the anomalous wave function of correlated quasiparticles in the ferromagnetic region. The solid lines correspond to electrons, and the dashed lines to holes; the arrows indicate the direction of the velocity.

$$
\begin{gathered}
S_{1}=-Q x-\alpha, \\
S_{2}=Q x-\alpha, \\
S_{3}=-Q\left(2 d_{f}+x\right)-\alpha, \\
S_{4}=Q\left(2 d_{f}+x\right)-\alpha
\end{gathered}
$$

(note that $x<0$ ), where $Q$ is the difference between the wave numbers of the electron and the hole, and $\alpha=\arccos (E / \Delta)$ is the phase of the Andreev reflection. To make our arguments more clear, we assume that the ferromagnet is strong, the SF interface is ideal, and consider the clean limit first: then $Q=k_{e}-k_{h}=\sqrt{2 m\left(E+E_{\mathrm{ex}}+\mu\right)}-\sqrt{2 m\left(-E-E_{\mathrm{ex}}+\mu\right)}$ $\approx 2 E_{\text {ex }} / v$, where $E$ is the quasiparticle energy, $\mu$ is the Fermi energy, and $v$ is the Fermi velocity. Thus the anomalous wave function of the quasiparticles is

$$
F(x) \propto \sum_{n=1}^{4} \exp \left(i S_{n}\right) \propto \cos \left(Q d_{f}\right) \cos \left(Q\left[d_{f}+x\right]\right) .
$$

The suppression of $T_{c}$ by the ferromagnet is determined by the value of the wave function at the SF interface: $F(0)$ $\propto \cos ^{2}\left(Q d_{f}\right)$. The minimum of $T_{c}$ corresponds to the minimum value of $F(0)$ which is achieved at $d_{f}=\pi / 2 Q$. In the dirty limit the above expression for $Q$ is replaced by

$$
Q=\sqrt{\frac{E_{\mathrm{ex}}}{D_{f}}} \equiv \frac{2 \pi}{\lambda_{\mathrm{ex}}}
$$

(here we have defined the wavelength of the oscillations $\left.\lambda_{\mathrm{ex}}\right)$; hence the minimum of $T_{c}\left(d_{f}\right)$ takes place at

$$
d_{f}^{(\min )}=\frac{\pi}{2} \sqrt{\frac{D_{f}}{E_{\mathrm{ex}}}}=\frac{\lambda_{\mathrm{ex}}}{4} .
$$

For the bilayer of Ref. 11 we obtain $d_{f}^{(\min )} \approx 7 \mathrm{~nm}$, whereas the experimental value is $5 \mathrm{~nm}$ (Fig. 2); thus our qualitative estimate is reasonable.

The arguments given above seem to yield not only the minimum but rather a succession of minima and maxima. However, numerically we obtain either a single minimum or a minimum followed by a weak maximum (Fig. 3). The reason for this is that actually the anomalous wave function not only oscillates in the ferromagnetic layer but also decays exponentially, which makes the amplitude of the subsequent oscillations almost invisible.

Finally, we note that our arguments concerning oscillations of $F(x)$ also apply to a half-infinite ferromagnet, where we should take into account only the trajectories 1 and 2 (see Fig. 7). This yields $F(x) \propto \cos (Q x)$ (another qualitative explanation of this result can be found, for example, in Ref. 14).

\section{B. Multilayered structures}

The methods developed and the results obtained in this paper apply directly to more complicated symmetric multilayered structures in the 0-state such as SFS and FSF trilayers, SFIFS and FSISF systems (I denotes an arbitrary potential barrier), and SF superlattices. In such systems an SF 
bilayer can be considered as a unit cell, and joining together the solutions of the Usadel equations in each bilayer we obtain the solution for the whole system (for more details see Sec. VIII of Ref. 28).

Our methods can be generalized to take account of possible superconductive and/or magnetic $\pi$ states (when $\Delta$ and/or $E_{\text {ex }}$ may change their signs from layer to layer). In this case the system cannot be equivalently separated into a set of bilayers. Mathematically, this means that the solutions of the Usadel equations lose their purely cosine form [see Eqs. (7), (14), (15), (19), (20), and (30b)] acquiring a sine part as well.

\section{Complex diffusion constant?}

Finally, we comment on Refs. 4, 15, 16, and 29, where the authors considered (in the vicinity of $T_{c}$ ) diffusion equations with a complex diffusion constant $D_{f}$ for the F part of the structure. This implies small complex corrections to $D_{f}$ over $E_{\text {ex }} \tau \ll 1$ in the Usadel equations ( $\tau$ is the time of the mean free path). However, we disagree with this method for the following reason: although the complex $D_{f}$ can indeed be formally obtained in the course of the standard derivation of the Usadel equations ${ }^{17}$ from the Eilenberger ones ${ }^{30}$ by expanding over the spherical harmonics, one can check that the higher harmonics neglected in the derivation have the same order of magnitude as the retained complex correction to $D_{f}$. Hence the complexity of $D_{f}$ in the context of the Usadel equations is the excess of accuracy. Below we present our arguments.

We give a brief derivation of the Usadel equations showing how the complex diffusion constant can be obtained and why this result cannot be trusted. In the "quasi-onedimensional" geometry (which means that the parameters vary only as a function of $x$ ) the linearized Eilenberger equation in the presence of disorder and the exchange field has the form

$$
\frac{v \cos \theta}{2} \frac{d}{d x} F+\left(\omega_{n}+\frac{1}{2 \tau}+i E_{\mathrm{ex}}\right) F=\Delta+\frac{\langle F\rangle}{2 \tau},
$$

where, for simplicity, we assume a positive Matsubara frequency $\omega_{n}>0$, and $\theta$ is the angle between the $x$ axis and the direction of the Fermi velocity $\mathbf{v}$, while $\langle\cdots\rangle$ denotes angular averaging over the spherical angles. The disorder is characterized by the time of the mean free path $\tau$ and the mean free path $l$ (to be used below). In the dirty limit the anomalous Green function $F$ is nearly isotropic. However, to obtain the Usadel equation for the isotropic part of $F$, we must also take into account the next term from the full Legendre polynomial expansion:

$$
\begin{aligned}
F\left(x, \omega_{n}, \theta\right) & =\sum_{k=0}^{\infty} F_{k}\left(x, \omega_{n}\right) P_{k}(\cos \theta) \\
& \approx F_{0}\left(x, \omega_{n}\right)+F_{1}\left(x, \omega_{n}\right) \cos \theta .
\end{aligned}
$$

Here we have neglected the harmonics with $k \geqslant 2$ assuming them small; we shall check this assumption later.
Averaging Eq. (42) over the spherical angles first directly and second after being multiplied by $\cos \theta$, we arrive at

$$
\begin{gathered}
\frac{v}{6} \frac{d}{d x} F_{1}+\left(\omega_{n}+i E_{\mathrm{ex}}\right) F_{0}=\Delta \\
\frac{v}{2} \frac{d}{d x} F_{0}+\left(\omega_{n}+\frac{1}{2 \tau}+i E_{\mathrm{ex}}\right) F_{1}=0
\end{gathered}
$$

Equation (45) yields

$$
F_{1}=-\left(\frac{l}{1+2 \omega_{n} \tau+2 i E_{\mathrm{ex}} \tau}\right) \frac{d}{d x} F_{0}
$$

then Eq. (44) leads to

$$
\begin{gathered}
\frac{D}{2} \frac{d^{2}}{d x^{2}} F_{0}-\left(\omega_{n}+i E_{\mathrm{ex}}\right) F_{0}+\Delta=0, \\
D=\frac{v l / 3}{1+2 \omega_{n} \tau+2 i E_{\mathrm{ex}} \tau} .
\end{gathered}
$$

Now we must check that the assumption $\left|F_{1} / F_{0}\right| \ll 1$, $\left|F_{2} / F_{1}\right| \ll 1$, etc. that we used is indeed satisfied. From Eq. (46) we obtain

$$
\left|\frac{F_{1}}{F_{0}}\right| \sim \frac{l / L}{\max \left(1,2 \omega_{n} \tau, 2 E_{\mathrm{ex}} \tau\right)},
$$

where $L$ is the characteristic space scale on which $F_{0}$ varies. According to the Usadel equation (47), it is given by

$$
L \sim \frac{l}{\sqrt{\max \left(1,2 \omega_{n} \tau, 2 E_{\mathrm{ex}} \tau\right) \max \left(2 \omega_{n} \tau, 2 E_{\mathrm{ex}} \tau\right)}},
$$

and the condition of the Usadel equation's validity is written as

$$
\left|\frac{F_{1}}{F_{0}}\right| \sim \sqrt{\frac{\max \left(2 \omega_{n} \tau, 2 E_{\mathrm{ex}} \tau\right)}{\max \left(1,2 \omega_{n} \tau, 2 E_{\mathrm{ex}} \tau\right)}} \ll 1
$$

[similarly, we can also keep the term with $k=2$ in series (43), which yields $\left|F_{2} / F_{1}\right| \sim\left|F_{1} / F_{0}\right|$, etc.].

Finally, condition (50) takes the form

$$
2 \pi T_{c s} \tau \ll 1, \quad 2 E_{\mathrm{ex}} \tau \ll 1
$$

(we have taken into account that the characteristic energy is $\left.\omega_{n} \sim \pi T_{c s}\right)$.

Now we can analyze our results. If condition (51) is satisfied and the Usadel equation is valid, the neglected angular harmonics have the relative order of magnitude $\left|F_{2} / F_{0}\right|$ $\sim \max \left(2 \pi T_{c s} \tau, 2 E_{\mathrm{ex}} \tau\right)$; hence we cannot retain the terms of the same order in the diffusion constant [see Eq. (47)], and we should use the standard expression $D=v l / 3$.

\section{CONCLUSIONS}

In the present paper we have developed two methods for calculating the critical temperature of a SF bilayer as a function of its parameters (the thicknesses and material param- 
eters of the layers, and the quality of the interface). The multimode method is a generalization of the corresponding approach developed in Ref. 20 for SN systems. However, the rigorous justification of this method is not clear. Therefore, we propose yet another approach - the method of fundamental solution, which is mathematically rigorous. The results demonstrate that the two methods are equivalent; however, at low temperatures (compared to $T_{c s}$ ) the accuracy requirements are stricter for the multimode method, and the method of fundamental solution is preferable. Comparing our method with experiment we obtain good agreement.

In the general case, we observe three characteristic types of $T_{c}\left(d_{f}\right)$ behavior: (1) a nonmonotonic decay of $T_{c}$ to a finite value exhibiting a minimum at particular $d_{f}$; (2) a reentrant behavior, characterized by vanishing of $T_{c}$ in a certain interval of $d_{f}$ and finite values otherwise, and (3) a monotonic decay of $T_{c}$ and vanishing at finite $d_{f}$. Qualitatively, the nonmonotonic behavior of $T_{c}\left(d_{f}\right)$ is explained by interference of quasiparticles in the $\mathrm{F}$ layer, which can be either constructive or destructive depending on the value of $d_{f}$.

Using the developed methods we have checked the accuracy of the widely used single-mode approximation. We conclude that although at some parameters the results of the single-mode and exact methods are close, in the general case they are quantitatively and even qualitatively different. Thus, to obtain reliable results one should use one of the exact (multimode or fundamental-solution) techniques. The spatial dependence of the order parameter (at the transition point) is shown to be almost insensitive to the value of $T_{c}$.

The methods developed and the results obtained in this paper apply directly to more complicated symmetric multilayered structures in the 0-state such as SFS and FSF trilayers, SFIFS and FSISF systems, and SF superlattices. Our methods can be generalized to take account of possible superconductive and/or magnetic $\pi$ states (when $\Delta$ and/or $E_{\mathrm{ex}}$ may change their signs from layer to layer).

We argue that the use of the complex diffusion constant in the Usadel equation is the excess of accuracy. In several limiting cases, $T_{c}$ is considered analytically.

\section{ACKNOWLEDGMENTS}

We thank V. V. Ryazanov and M. V. Feigel'man for stimulating discussions. We are especially indebted to V. V. Ryazanov for communicating the experimental result of his group to us prior to the detailed publication. We are also grateful to J. Aarts, A. I. Buzdin, M. Yu. Kupriyanov, Yu. Oreg, and L. R. Tagirov for useful comments. Ya.V.F. acknowledges financial support from the Russian Foundation for Basic Research (RFBR) under Project No. 01-02-17759, and from Forschungszentrum Jülich (Landau Scholarship). The research of N.M.C. was supported by the RFBR (Projects Nos. 01-02-06230 and 00-02-16617), by Forschungszentrum Jülich (Landau Scholarship), by the Netherlands Organization for Scientific Research (NWO), by the Einstein Center, and by the Swiss National Foundation.

\section{APPENDIX A: ANALYTICAL RESULTS FOR A THIN S LAYER}

(i) When $d_{s} \ll \xi_{s}$ and $E_{\text {ex }} \gg \pi T_{c s}$, problems (10)-(13) can be solved analytically. The first of the above conditions implies that $\Delta$ can be considered constant, and $F^{+}$weakly depends on the spatial coordinate; so $F^{+}\left(x, \omega_{n}\right)=2 \Delta / \omega_{n}$ $+A\left(\omega_{n}\right) \cosh \left(k_{s}\left[x-d_{s}\right]\right)$. The boundary conditions determine the coefficient $A$; as a result,

$F^{+}\left(\omega_{n}\right) \equiv F^{+}\left(x=0, \omega_{n}\right)=\frac{2 \Delta}{\omega_{n}}\left[\frac{A_{s}\left(\omega_{n}\right)}{A_{s}\left(\omega_{n}\right)+W\left(\omega_{n}\right)}\right]$,

where $k_{s}, A_{s}$, and $W$ are defined in Eq. (12). Finally, the self-consistency equation for $T_{c}$ takes the form

$$
\ln \frac{T_{c s}}{T_{c}}=\operatorname{Re} \psi\left(\frac{1}{2}+\frac{\gamma}{2} \frac{\xi_{s}}{d_{s}} \frac{1}{\gamma_{b}+B_{f}} \frac{T_{c s}}{T_{c}}\right)-\psi\left(\frac{1}{2}\right),
$$

where $B_{f}$ does not depend on $\omega_{n}$ due to the condition $E_{\mathrm{ex}}$ $\gg \pi T_{c s}$ :

$$
B_{f}=\left[k_{f} \xi_{f} \tanh \left(k_{f} d_{f}\right)\right]^{-1}, \quad k_{f}=\frac{1}{\xi_{f}} \sqrt{\frac{i E_{\mathrm{ex}}}{\pi T_{c s}}} .
$$

(ii) If the $\mathrm{F}$ layer is also thin, $d_{f} \ll \sqrt{D_{f} / 2 E_{\text {ex }}}$, Eq. (A2) is further simplified,

$$
\ln \frac{T_{c s}}{T_{c}}=\operatorname{Re} \psi\left(\frac{1}{2}+\frac{\tau_{f}}{\tau_{s}}\left[\frac{1}{-i+\tau_{f} E_{\mathrm{ex}}}\right] \frac{E_{\mathrm{ex}}}{2 \pi T_{c}}\right)-\psi\left(\frac{1}{2}\right),
$$

where $\tau_{s}$ and $\tau_{f}$ are defined similarly to Ref. 28,

$$
\tau_{s}=\frac{2 d_{s} R_{b} \mathcal{A}}{\rho_{s} D_{s}}, \quad \tau_{f}=\frac{2 d_{f} R_{b} \mathcal{A}}{\rho_{f} D_{f}},
$$

and have the physical meaning of the escape time from the corresponding layer. They are related to the quantities $\gamma$ and $\gamma_{b}$ used in the body of the paper as

$$
\tau_{s}=\frac{\gamma_{b}}{\gamma} \frac{1}{\pi T_{c s}} \frac{d_{s}}{\xi_{s}}, \quad \tau_{f}=\gamma_{b} \frac{1}{\pi T_{c s}} \frac{d_{f}}{\xi_{f}} .
$$

(iii) If the $\mathrm{S}$ layer is thin, $d_{s} \ll \xi_{s}$, and the SF interface is opaque, $\gamma_{b} \rightarrow \infty$, the critical temperature of the bilayer only slightly deviates from $T_{c s}$. In this limit Eq. (A1) applies with $W=\gamma / \gamma_{b} \ll 1$, and we finally obtain

$$
T_{c}=T_{c s}-\frac{\pi}{4 \tau_{s}} .
$$

Interestingly, characteristics of the $\mathrm{F}$ layer $\left(d_{f}, E_{\text {ex }}\right.$, etc.) do not enter the formula. In particular, this formula is valid for an $\mathrm{SN}$ bilayer $^{31,32}$ (where $\mathrm{N}$ is a nonmagnetic normal material, $E_{\mathrm{ex}}=0$ ) because Eq. (A7) was obtained without any assumptions about the value of the exchange energy.

\section{Transparent interface}

When both layers are very thin $\left[d_{s} \ll \sqrt{D_{s} / 2 \omega_{D}}\right.$, $d_{f} \ll \min \left(\sqrt{D_{f} / 2 \omega_{D}}, \sqrt{D_{f} / 2 E_{\text {ex }}}\right)$, with $\omega_{D}$ the Debye energy 
of the $S$ metal] and the interface is transparent, the bilayer is equivalent to a homogeneous superconducting layer with internal exchange field. This layer is described by effective parameters: the pairing potential $\Delta^{\text {(eff) }}$, the exchange field $E_{\mathrm{ex}}^{\text {(eff) }}$, and the pairing constant $\lambda^{\text {(eff) }}$. In this subsection we develop the ideas of Ref. 33, demonstrate a simple derivation of this description, and find the limits of its applicability.

The Usadel equations (1) and (2) for the two layers can be written as a single equation,

$$
\begin{aligned}
& \frac{D_{f} \theta(-x)+D_{s} \theta(x)}{2} \frac{d^{2} F}{d x^{2}} \\
& -\left|\omega_{n}\right| F-i E_{\mathrm{ex}} \operatorname{sgn}\left(\omega_{n}\right) \theta(-x) F+\Delta \theta(x)=0,
\end{aligned}
$$

where $\theta$ is the Heaviside function $[\theta(x>0)=1, \theta(x<0)$ $=0]$. The self-consistency equation (3) can be rewritten as

$$
\Delta(x)=\lambda \theta(x) \pi T \sum_{\omega_{n}} F\left(x, \omega_{n}\right),
$$

where $\lambda$ is the pairing constant.

First, we consider the ideal SF interface: $\gamma_{b}=0$ [see Eq. (6)], then $F(x)$ is continuous at the interface and nearly constant across the whole bilayer, i.e., $F_{s}(x) \approx F_{f}(x)=F$. Applying the integral operator to Eq. (A8),

$$
\frac{\nu_{f}}{\nu_{s} d_{s}+\nu_{f} d_{f}} \int_{-d_{f}}^{0} d x+\frac{\nu_{s}}{\nu_{s} d_{s}+\nu_{f} d_{f}} \int_{0}^{d_{s}} d x
$$

(here $\nu$ is the normal-metal density of states), and cancelling gradient terms due to boundary condition (5), we obtain the equations describing a homogeneous layer,

$$
\begin{gathered}
-\left|\omega_{n}\right| F\left(\omega_{n}\right)-i E_{\mathrm{ex}}^{(\mathrm{eff})} \operatorname{sgn}\left(\omega_{n}\right) F\left(\omega_{n}\right)+\Delta^{(\mathrm{eff})}=0, \\
\Delta^{(\mathrm{eff})}=\lambda^{(\mathrm{eff})} \pi T \sum_{\omega_{n}} F\left(\omega_{n}\right),
\end{gathered}
$$

with the effective parameters (see also Ref. 33)

$$
\begin{gathered}
E_{\mathrm{ex}}^{(\mathrm{eff})}=\frac{\tau_{f}}{\tau_{s}+\tau_{f}} E_{\mathrm{ex}}, \quad \Delta^{(\mathrm{eff})}=\frac{\tau_{s}}{\tau_{s}+\tau_{f}} \Delta, \\
\lambda^{(\mathrm{eff})}=\frac{\tau_{s}}{\tau_{s}+\tau_{f}} \lambda, \quad T_{c s}^{(\mathrm{eff})}=\frac{\gamma_{E}}{\pi} 2 \omega_{D} \exp \left(-\frac{1}{\lambda^{(\mathrm{eff})}}\right),
\end{gathered}
$$

where $\gamma_{E}$ is Euler's constant, and $T_{c s}^{(\mathrm{eff})}$ is the critical temperature of the layer in the absence of ferromagnetism (i.e., at $E_{\mathrm{ex}}^{(\mathrm{eff})}=0$ ). The critical temperature is determined by the equation

$$
\ln \frac{T_{c s}^{(\mathrm{eff})}}{T_{c}}=\operatorname{Re} \psi\left(\frac{1}{2}+i \frac{E_{\mathrm{ex}}^{(\mathrm{eff})}}{2 \pi T_{c}}\right)-\psi\left(\frac{1}{2}\right) .
$$

Actually, the description in terms of effective parameters (A13) is applicable at an arbitrary temperature (i.e., when the Usadel equations are nonlinear) and has a clear physical in- terpretation: the superconducting $(\Delta, \lambda)$ and ferromagnetic $\left(E_{\mathrm{ex}}\right)$ parameters are renormalized according to the part of time spent by quasiparticles in the corresponding layer. This physical picture is based on interpretation of $\tau$ as escape times, which we present in Appendix A 2.

Now we discuss the applicability of the above description for a nonideal interface $\left(\gamma_{b} \neq 0\right)$. In this case $F$ is nearly constant in each layer, but these constants are different: $F_{s}(x) \approx F_{s}+C_{s}\left(x-d_{s}\right)^{2}, \quad F_{f}(x) \approx F_{f}+C_{f}\left(x+d_{f}\right)^{2}$, where $\left|F_{s}\right| \gg\left|C_{s}\right| d_{s}^{2}$ and $\left|F_{f}\right| \gg\left|C_{f}\right| d_{f}^{2}$. Using the Usadel equation (A8) and boundary conditions (5) and (6), we find the difference $\delta F \equiv F_{s}-F_{f}$ :

$$
\delta F=\frac{\Delta}{\frac{1}{\tau_{s}}+\left|\omega_{n}\right|\left[1+\frac{1}{\tau_{f}\left(\left|\omega_{n}\right|+i E_{\mathrm{ex}} \operatorname{sgn} \omega_{n}\right)}\right]} .
$$

Finally, the homogeneous description is valid when $|\delta F / F|$ $\ll 1$ [with $F$ determined by Eq. (A11)], which yields

$$
\max \left(E_{\text {ex }}, \omega_{D}\right) \max \left(\tau_{s}, \tau_{f}\right) \ll 1
$$

(here $\omega_{n} \sim \omega_{D}$ has been taken as the largest characteristic energy scale in the quasihomogeneous bilayer).

\section{Interpretation of $\tau$ as escape times}

The quantities $\tau_{s}$ and $\tau_{f}$ introduced in Eq. (A5) may be interpreted as escape times from the corresponding layers. The arguments go as follows.

If the layers are thin, then the diffusion inside the layers is "fast" and the escape time from a layer is determined by the interface resistance. The time of penetration through a layer or the interface is determined by the corresponding resistance $R_{s(f)}$ or $R_{b}$, hence the diffusion is "fast" if $R_{s(f)} \ll R_{b}$.

Let us use the detailed balance approach, and consider an interval of energy $d E$. In the $\mathrm{S}$ layer, the charge in this interval is $Q_{s}=e \nu_{s} d E \mathcal{A} d_{s}$. Let us define the escape time from the $\mathrm{S}$ layer $t_{s}$, so that the current from $\mathrm{S}$ to $\mathrm{F}$ is equal to $Q_{s} / t_{s}$. On the other hand, this current can be written as $d E / e R_{b}$; hence

$$
\frac{Q_{s}}{t_{s}}=\frac{d E}{e R_{b}},
$$

and we immediately obtain

$$
t_{s}=\frac{d_{s} R_{b} \mathcal{A}}{\rho_{s} D_{s}} .
$$

Similarly, we obtain the expression for the escape time from the $\mathrm{F}$ layer $t_{f}$. As a result, the relations between the quantities $\tau$, defined in Eq. (A5), and the escape times $t$ are simply

$$
\tau_{s}=2 t_{s}, \quad \tau_{f}=2 t_{f} .
$$

Microscopic expressions for the escape times may be obtained using the Sharvin formula for the interface resistance. Assuming, for definiteness, that the Fermi velocity is smaller in the $\mathrm{S}$ metal, $v_{s}<v_{f}$, we obtain 


$$
R_{b}=\frac{\pi r_{b}}{e^{2} \nu_{s} v_{s} \mathcal{A}}
$$

and consequently

$$
t_{s}=\pi \frac{d_{s}}{v_{s}} r_{b}, \quad t_{f}=\pi \frac{v_{f} d_{f}}{v_{s}^{2}} r_{b},
$$

where $r_{b}$ is the inverse transparency of one channel. The asymmetry in these expressions stems from our assumption $v_{s}<v_{f}$. In the opposite case the indices $s$ and $f$ in Eqs. (A20) and (A21) should be interchanged.

\section{APPENDIX B: \\ APPLICABILITY OF THE SINGLE-MODE APPROXIMATION}

As pointed out in Sec. III A, the single-mode approximation (SMA) is applicable only if the parameters are such that $W$ [see Eq. (12)] can be considered $\omega_{n}$ independent. An example is the case when $\gamma_{b} \gg\left|B_{f}\right|$, hence $W=\gamma / \gamma_{b}$.

The condition $\gamma_{b} \gg\left|B_{f}\right|$ can be written in a simpler form; to this end we should estimate $\left|B_{f}\right|$. We introduce the real and imaginary parts of $k_{f}, k_{f}=k_{f}^{\prime}+i k_{f}^{\prime \prime}$, and note that $k_{f}^{\prime}$ $>k_{f}^{\prime \prime}$. Then using the properties of the trigonometric functions and the estimate $\tanh x \sim \min (1, x)$ we obtain

$$
\left|B_{f}\right| \sim\left[k_{f}^{\prime} \xi_{f} \tanh \left(k_{f}^{\prime} d_{f}\right)\right]^{-1},
$$

and finally cast the condition $\gamma_{b} \gg\left|B_{f}\right|$ into the form

$$
\frac{1}{\gamma_{b}} \ll \min \left\{\sqrt{\max \left(\frac{T_{c}}{T_{c s}}, \frac{E_{\mathrm{ex}}}{\pi T_{c s}}\right)} ; \frac{d_{f}}{\xi_{f}} \max \left(\frac{T_{c}}{T_{c s}}, \frac{E_{\mathrm{ex}}}{\pi T_{c s}}\right)\right\},
$$

where the ratio $T_{c} / T_{c s}$ originates from $\omega_{n} / \pi T_{c s}$ with $\omega_{n}$ $\sim \pi T_{c}$ as the characteristic energy scale in the bilayer.

If condition (B2) is satisfied, then the SMA is valid and $T_{c}$ is determined by the equations

$$
\begin{aligned}
\ln \frac{T_{c s}}{T_{c}}=\psi\left(\frac{1}{2}+\frac{\Omega^{2}}{2} \frac{T_{c s}}{T_{c}}\right)-\psi\left(\frac{1}{2}\right), \\
\Omega \tan \left(\Omega \frac{d_{s}}{\xi_{s}}\right)=\frac{\gamma}{\gamma_{b}} .
\end{aligned}
$$

These equations can be further simplified in two limiting cases which we consider below.

$$
\text { (1) } \frac{\gamma}{\gamma_{b}} \frac{d_{s}}{\xi_{s}} \ll 1:
$$

In this case Eq. (B4) yields $\Omega^{2}=\left(\gamma / \gamma_{b}\right)\left(\xi_{s} / d_{s}\right)$, and Eq. (B3) takes the form

$$
\ln \frac{T_{c s}}{T_{c}}=\psi\left(\frac{1}{2}+\frac{1}{2} \frac{\gamma}{\gamma_{b}} \frac{\xi_{s}}{d_{s}} \frac{T_{c s}}{T_{c}}\right)-\psi\left(\frac{1}{2}\right),
$$

which reproduces the $\gamma_{b} \gg\left|B_{f}\right|$ limit of Eq. (A2).

$$
\text { (2) } \frac{\gamma}{\gamma_{b}} \frac{d_{s}}{\xi_{s}} \gg 1 \text { : }
$$

In this case Eq. (B4) yields $\Omega d_{s} / \xi_{s}=\pi / 2$, and Eq. (B3) takes the form

$$
\ln \frac{T_{c s}}{T_{c}}=\psi\left(\frac{1}{2}+\frac{\pi^{2}}{8}\left[\frac{\xi_{s}}{d_{s}}\right]^{2} \frac{T_{c s}}{T_{c}}\right)-\psi\left(\frac{1}{2}\right) .
$$

Equations (B3)-(B6) can be used for calculating the critical temperature $T_{c}$ and the critical thickness of the $\mathrm{S}$ layer $d_{s}^{(\text {cr) }}$ below which the superconductivity in the SF bilayer vanishes (i.e., $T_{c}=0$ ).

\section{Results for the critical temperature}

In the limit when $T_{c}$ is close to $T_{c s}$, Eqs. (B5) and (B6) yield

$$
T_{c}=T_{c s}\left(1-\frac{\pi^{2}}{4} \frac{\gamma}{\gamma_{b}} \frac{\xi_{s}}{d_{s}}\right) \quad \text { if } \frac{\gamma}{\gamma_{b}} \ll \min \left(\frac{d_{s}}{\xi_{s}}, \frac{\xi_{s}}{d_{s}}\right),
$$

and

$$
T_{c}=T_{c s}\left[1-\left(\frac{\pi^{2}}{4} \frac{\xi_{s}}{d_{s}}\right)^{2}\right] \quad \text { if } \frac{d_{s}}{\xi_{s}} \gg \max \left(1, \frac{\gamma_{b}}{\gamma}\right) .
$$

Using relations (A6) one can check that result (B7) is equivalent to Eq. (A7).

\section{Results for the critical thickness}

The critical thickness of the S layer $d_{s}^{(\mathrm{cr})}$ is defined as the thickness below which there is no superconductivity in the SF bilayer: $T_{c}\left(d_{s}^{\text {(cr) }}\right)=0$. When $T_{c} \rightarrow 0$, Eq. (B3) yields $\Omega$ $=1 / \sqrt{2 \gamma_{E}}$ (where $\gamma_{E} \approx 1.78$ is Euler's constant), and Eq. (B4) takes the form

$$
\frac{1}{\sqrt{2 \gamma_{E}}} \tan \left(\frac{1}{\sqrt{2 \gamma_{E}}} \frac{d_{s}^{(\mathrm{cr})}}{\xi_{s}}\right)=\frac{\gamma}{\gamma_{b}}
$$

Explicit results for $d_{s}^{(\mathrm{cr})}$ can be obtained in limiting cases,

$$
\frac{d_{s}^{(\mathrm{cr})}}{\xi_{s}}=2 \gamma_{E} \frac{\gamma}{\gamma_{b}} \quad \text { if } \frac{\gamma}{\gamma_{b}} \frac{d_{s}}{\xi_{s}} \ll 1
$$

and

$$
\frac{d_{s}^{(\mathrm{cr})}}{\xi_{s}}=\pi \sqrt{\frac{\gamma_{E}}{2}} \quad \text { if } \frac{\gamma}{\gamma_{b}} \frac{d_{s}}{\xi_{s}} \gg 1
$$

\section{APPENDIX C: \\ SPATIAL DEPENDENCE OF THE ORDER PARAMETER}

According to the self-consistency equation, in the $\mathrm{S}$ layer the order parameter $F(x, \tau=0)$ is proportional to $\Delta(x)$,

$$
F_{s}(x, \tau=0)=\frac{\Delta(x)}{\pi \lambda},
$$


where $\lambda$ is the pairing constant which can be expressed via the Debye energy:

$$
\lambda^{-1}=\ln \left(\frac{2 \gamma_{E} \omega_{D}}{\pi T_{c s}}\right) .
$$

The pairing potential $\Delta(x)$ can be found as the eigenvector of the matrix $\hat{L}-\hat{1} \ln \left(T_{c s} / T_{c}\right)$ [see Eq. (33)], corresponding to the zero eigenvalue.

After that we can express $F(x, \tau=0)$ in the $\mathrm{F}$ layer via $\Delta(x)$ in the superconductor. The Green function $F_{f}\left(x, \omega_{n}\right)$ in the F layer is given by Eq. (7), with $C\left(\omega_{n}\right)$ found from the boundary conditions:

$$
C\left(\omega_{n}\right)=\left(\frac{B_{f}}{\gamma_{b}+B_{f}}\right) \frac{F_{s}\left(0, \omega_{n}\right)}{\cosh \left(k_{f} d_{f}\right)} .
$$

The Green function at the S side of the SF interface is

$$
F_{s}\left(0, \omega_{n}\right)=\frac{F_{s}^{+}\left(0, \omega_{n}\right)+F_{s}^{-}\left(0, \omega_{n}\right)}{2} .
$$

The symmetric part $F_{s}^{+}$is given by Eq. (31). The antisymmetric part is

$$
F_{s}^{-}=C^{-}\left(\omega_{n}\right) \cosh \left(k_{s}\left[x-d_{s}\right]\right),
$$

with $C^{-}\left(\omega_{n}\right)$ found from the boundary conditions,

$$
C^{-}\left(\omega_{n}\right)=\left[\frac{i \gamma \operatorname{Im} B_{f}}{A_{s}\left|\gamma_{b}+B_{f}\right|^{2}+\gamma\left(\gamma_{b}+\operatorname{Re} B_{f}\right)}\right] \frac{F_{s}^{+}\left(0, \omega_{n}\right)}{\cosh \left(k_{s} d_{s}\right)} .
$$

Finally, the order parameter in the $\mathrm{F}$ layer is the Fourier transform [see Eq. (34)] of

$$
\begin{aligned}
F_{f}\left(x, \omega_{n}\right)= & {\left[1+\frac{i \gamma \operatorname{Im} B_{f}}{A_{s}\left|\gamma_{b}+B_{f}\right|^{2}+\gamma\left(\gamma_{b}+\operatorname{Re} B_{f}\right)}\right] } \\
& \times\left(\frac{B_{f}}{\gamma_{b}+B_{f}}\right) \frac{\cosh \left(k_{f}\left[x+d_{f}\right]\right)}{\cosh \left(k_{f} d_{f}\right)} \\
& \times \int_{0}^{d_{s}} G\left(0, y ; \omega_{n}\right) \Delta(y) d y .
\end{aligned}
$$

*Electronic address: fominov@landau.ac.ru

†Electronic address: nms@landau.ac.ru

Electronic address: a.golubov@tn.utwente.nl

${ }^{1}$ V. V. Ryazanov, V. A. Oboznov, A. Yu. Rusanov, A. V. Veretennikov, A. A. Golubov, and J. Aarts, Phys. Rev. Lett. 86, 2427 (2001); V. V. Ryazanov, V. A. Oboznov, A. V. Veretennikov, and A. Yu. Rusanov, Phys. Rev. B 65, 020501(R) (2001).

2 T. Kontos, M. Aprili, J. Lesueur, and X. Grison, Phys. Rev. Lett. 86, 304 (2001); T. Kontos, M. Aprili, J. Lesueur, F. Genêt, B. Stephanidis, and R. Boursier, cond-mat/0201104 (unpublished).

${ }^{3}$ Z. Radović, M. Ledvij, Lj. Dobrosavljević-Grujić, A. I. Buzdin, and J. R. Clem, Phys. Rev. B 44, 759 (1991).

${ }^{4}$ L. R. Tagirov, Phys. Rev. Lett. 83, 2058 (1999).

${ }^{5}$ A. Buzdin, Phys. Rev. B 62, 11377 (2000).

${ }^{6}$ M. Zareyan, W. Belzig, and Yu. V. Nazarov, Phys. Rev. Lett. 86, 308 (2001).

${ }^{7}$ J. S. Jiang, D. Davidović, D. H. Reich, and C. L. Chien, Phys. Rev. Lett. 74, 314 (1995).

${ }^{8}$ Th. Mühge, N. N. Garif'yanov, Yu. V. Goryunov, G. G. Khaliullin, L. R. Tagirov, K. Westerholt, I. A. Garifullin, and H. Zabel, Phys. Rev. Lett. 77, 1857 (1996).

${ }^{9}$ J. Aarts, J. M. E. Geers, E. Brück, A. A. Golubov, and R. Coehoorn, Phys. Rev. B 56, 2779 (1997).

${ }^{10}$ L. Lazar, K. Westerholt, H. Zabel, L. R. Tagirov, Yu. V. Goryunov, N. N. Garif'yanov, and I. A. Garifullin, Phys. Rev. B 61, 3711 (2000).

${ }^{11}$ V. V. Ryazanov et al. (unpublished).

${ }^{12}$ A. Rusanov, R. Boogaard, M. Hesselberth, H. Sellier, and J. Aarts, Physica C 369, 300 (2002).

${ }^{13}$ A. I. Buzdin, B. Vujičić, and M. Yu. Kupriyanov, Zh. Éksp. Teor. Fiz. 101, 231 (1992) [Sov. Phys. JETP 74, 124 (1992)].

${ }^{14}$ E. A. Demler, G. B. Arnold, and M. R. Beasley, Phys. Rev. B 55, 15174 (1997).

15 Yu. N. Proshin and M. G. Khusainov, Zh. Éksp. Teor. Fiz. 113, 1708 (1998) [JETP 86, 930 (1998)]; 116, 1887 (1999) [89, 1021
(1999)]. The same results are published more briefly in M. G. Khusainov and Yu. N. Proshin, Phys. Rev. B 56, R14 283 (1997); 62, 6832 (2000).

${ }^{16}$ L. R. Tagirov, Physica C 307, 145 (1998).

${ }^{17}$ K. D. Usadel, Phys. Rev. Lett. 25, 507 (1970).

${ }^{18}$ A. I. Larkin and Yu. N. Ovchinnikov, in Nonequilibrium Superconductivity, edited by D. N. Langenberg and A. I. Larkin (Elsevier, New York, 1986), p. 530, and references therein.

${ }^{19}$ J. Rammer and H. Smith, Rev. Mod. Phys. 58, 323 (1986).

${ }^{20}$ A. A. Golubov, M. Yu. Kupriyanov, V. F. Lukichev, and A. A. Orlikovskii, Mikroelektronika 12, 355 (1983) [Sov. J. Microelectron. 12, 191 (1984)].

${ }^{21}$ Ya. V. Fominov, N. M. Chtchelkatchev, and A. A. Golubov, Pis'ma Zh. Éksp. Teor. Fiz. 74, 101 (2001) [JETP Lett. 74, 96 (2001)].

${ }^{22}$ M. Yu. Kupriyanov and V. F. Lukichev, Zh. Éksp. Teor. Fiz. 94, 139 (1988) [Sov. Phys. JETP 67, 1163 (1988)].

${ }^{23}$ M. Abramowitz and I. A. Stegun, Handbook of Mathematical Functions (Dover, New York, 1974).

${ }^{24}$ P. M. Morse and H. Feshbach, Methods of Theoretical Physics (McGraw-Hill, New York, 1953), Vol. 1.

${ }^{25}$ V. V. Ryazanov (private communication).

${ }^{26}$ G. Sarma, J. Phys. Chem. Solids 24, 1029 (1963); see also D. Saint-James, G. Sarma, and E. J. Thomas, Type II Superconductivity (Pergamon, Oxford, 1969), p. 159.

${ }^{27}$ R. P. Feynman and A. R. Hibbs, Quantum Mechanics and Path Integrals (McGraw-Hill, New York, 1965).

${ }^{28}$ Ya. V. Fominov and M. V. Feigel'man, Phys. Rev. B 63, 094518 (2001). The quantities $\tau_{S}, \tau_{N}, R_{\text {int }}$, and $\rho_{\text {int }}$ used there are equivalent, respectively, to the quantities $\tau_{s}, \tau_{f}, R_{b}$, and $r_{b}$ from the present paper.

${ }^{29}$ I. Baladié and A. Buzdin, Phys. Rev. B 64, 224514 (2001).

${ }^{30}$ G. Eilenberger, Z. Phys. 214, 195 (1968).

${ }^{31}$ W. L. McMillan, Phys. Rev. 175, 537 (1968). The equations obtained by McMillan in the framework of the tunneling Hamil- 
tonian method were later derived microscopically (from the Usadel equations) by A. A. Golubov and M. Yu. Kupriyanov, Physica C 259, 27 (1996).

${ }^{32}$ To avoid confusion, we note that Eq. (39) from McMillan's paper (Ref. 31) determining the critical temperature of a SN bilayer is incorrect. The correct equation, following from Eqs. (37), (38), (40), and leading to Eq. (41) of Ref. 31, reads $\ln \left(T_{C S} / T_{C}\right)$ $=\left(\Gamma_{S} / \Gamma\right)\left[\psi\left(\frac{1}{2}+\Gamma / 2 \pi T_{C}\right)-\psi\left(\frac{1}{2}\right)\right]$.

${ }^{33}$ F. S. Bergeret, A. F. Volkov, and K. B. Efetov, Phys. Rev. Lett. 86, 3140 (2001); Phys. Rev. B 64, 134506 (2001). 